\title{
Pl2 | Quantum Crystallographic Studies Of Advanced Materials
}

Brummerstedt Iversen, Bo (Aarhus University, Aarhus, DNK)

Layered (2D) materials exhibit a variety of extraordinary properties, and recent focus has included topological insulators, electrode materials, monolayers, hetero structures - and thermoelectrics. The physical properties such as band gap or thermal and electrical conductivity are related to the detailed structural characteristics as well as the specific chemical bonding both within the covalent layers and across the van der Waal gap. It is generally assumed that layered materials exhibit anisotropic properties, but the properties are rarely discussed in direct relation to the specific chemical bonding characteristics of the solid.

Using advanced crystallographic analysis such as charge density modelling, NXMEM analysis, 3D-PDF as well as ab initio theoretical calculations, we have studied the crystal structures, chemical bonding and physical properties of a range of important thermoelectric materials including Cu2Se [1], Mg3Sb2 [2], SnS2 [3], TiS2 [4], SnSe [5] and PbS [6].

[1] a) E. Eikeland et al., IUCr-J 2017, 4, 467-485, b) K. J. Dalgaard et al., Adv. Theory Simul. 2018, 1, 1800068, N. Roth \& B. B. Iversen, Acta Crystallogr. Sect. A 2019, 75, 465-473

[2] J. Zhang et al., Nature Commun. 2016, 7, 10892; Nature Commun. 2017, 8, 13901; Nature Commun. 2018, 9, 4716; npj comp. mater. 2019, in press

[3] M. Fils $\varnothing$ et al., Dalton Trans. 2016, 45, $3798-3805$

[4] H. Kasai et al., Nature Materials 2018, 17, 249-252

[5] M. Sist et al., Acta Crystallogr. Sect. B. 2016, 72, 310-316

[6] C. Zeuthen et al., J. Am. Chem. Soc. 2019, 141,20, 8146-8157 